University of Wollongong

Research Online

Faculty of Engineering and Information

Faculty of Engineering and Information

Sciences - Papers: Part A

Sciences

January 2014

QoE-based network-centric resource allocation for on-demand uplink adaptive HTTP streaming over LTE network

Farhan Pervez

COMSATS Institute of Information Technology

Muhammad Salman Raheel

University of Wollongong, msr949@uowmail.edu.au

Follow this and additional works at: https://ro.uow.edu.au/eispapers

Research Online is the open access institutional repository for the University of Wollongong. For further information contact the UOW Library: research-pubs@uow.edu.au 


\title{
QoE-based network-centric resource allocation for on-demand uplink adaptive HTTP streaming over LTE network
}

\author{
Abstract \\ With the advent of community portals and video sharing websites, production and consumption of \\ multimedia content has increased tremendously. It is because of excellent video recording capabilities of \\ cameras available with mobile devices, and enhanced data rates offered by cellular networks, that the \\ uploading of high quality multimedia content is more realistic. Nowadays most of the video traffic is \\ HTTP-based, that provides rate adaptive streaming, known as Dynamic Adaptive Streaming over HTTP \\ (DASH). In this work we generate DASH video streams that can be supported on LTE uplink and then \\ assign resources to the video producers such that the QoE of on-demand viewers is maximized.

\section{Keywords} \\ allocation, demand, uplink, adaptive, qoe, http, Ite, streaming, network, over, centric, resource

\section{Publication Details} \\ F. Pervez \& M. Salman. Raheel, "QoE-based network-centric resource allocation for on-demand uplink \\ adaptive HTTP streaming over LTE network," in Application of Information and Communication \\ Technologies (AICT), 2014 IEEE 8th International Conference on, 2014, pp. 1-5.
}




\section{QoE-based Network-Centric Resource Allocation for On-demand Uplink Adaptive HTTP Streaming over LTE Network}

\author{
First Author: Farhan Pervez \\ Department of Electrical Engineering \\ COMSATS Institute of Information Technology \\ Lahore, Pakistan \\ farhanpervez@ciitlahore.edu.pk
}

\author{
Second Author: Muhammad Salman Raheel \\ Department of Engineering \\ University of Wollongong \\ New South Wales, Australia \\ msr949@uowmail.edu.au
}

\begin{abstract}
With the advent of community portals and video sharing websites, production and consumption of multimedia content has increased tremendously. It is because of excellent video recording capabilities of cameras available with mobile devices, and enhanced data rates offered by cellular networks, that the uploading of high quality multimedia content is more realistic. Nowadays most of the video traffic is HTTP-based, that provides rate adaptive streaming, known as Dynamic Adaptive Streaming over HTTP (DASH). In this work we generate DASH video streams that can be supported on LTE uplink and then assign resources to the video producers such that the QoE of on-demand viewers is maximized.
\end{abstract}

Keywords - DASH (Dynamic Adaptive Streaming over HTTP), QoE (Quality of Experience), resource management, Uplink

\section{INTRODUCTION}

4th generation (4G) mobile networks based on 3GPP LTE [1] provide high data rates for mobile users which enable them to use high quality Internet services. According to traffic and services forecasts, mobile video traffic in 2017 will exceed $66 \%$ of all generated traffic which will increase 26-fold compared to 2012 [2]. A large amount of this video traffic will be user generated. Some of the applications that are source for user generated content (UGC) are for example a car on a road monitoring its surroundings and sharing the content through mobile radio network, a camera used in an operating room for haptics communication between a doctor and his patient, and a mobile user recording and uploading videos of a live football match. A large share of video traffic nowadays is HTTP-based [3], that provides dynamic rate adaptive streaming. There has been efforts, largely by 3rd Generation Partnership Project (3GPP), to standardize this HTTP-based streaming for mobile networks, known as Dynamic Adaptive Streaming over HTTP
(3GP-DASH). In the near future it is expected that this type of dynamic adaptive streaming will also be a large share of the uplink traffic with LTE networks providing the data rates to support it. In this paper we first take into account the challenges that can be faced for the video producers and generate DASH video streams that can be supported on LTE uplink air interface. Then, we design an algorithm that allocates physical resources to the video producers such that the QoE for the on-demand viewers is maximized. Simulation results are presented for LTE uplink resource distribution that demonstrate the significance of the proposed optimization on the overall user experience. To the best of knowledge this work is one among the first in the direction of assigning optimized resources to DASH video producers over uplink.

The rest of the paper is organized as follows. In the next section, we first generate DASH video streams using a suitable video encoding scheme for uplink and then provide a discussion of related work. In Section III, we present our network-centric system. Section IV describes the LTE uplink model and the resource allocation framework for LTE uplink. Section V presents the DASH rate adaptation algorithm along with simulation results and Section VI concludes the paper.

\section{VIDEO ENCODING FOR UPLINK DASH}

\section{A. Generating DASH Video Streams}

According to the principle of the 3GP-DASH specification [4], the media is generated in video segments of specific duration at different bit rates. The client then HTTP request's the generated segments of different bit rates according to its network conditions. There are different ways of providing multiple bitrate representations of the media segments that need to be send from the producer to the viewer. One method is Advanced 
Video Coding (H.264/AVC) [5], in which a segment is completely and independently encoded at different bitrates. This can be done using a single layer codec. Another method is by encoding a segment in multiple layers by using Scalable Video Coding (SVC) [6]. This allows to store layers of the video as different representations which are additive to each other. In respect to AVC this type of encoding is referred as SVC extension of H.264/AVC.

Largely, the segments that are available at different bit rates are generated using H.264/AVC. As H.264/AVC results in a single-layer so this means that the encoded bit stream does not provide several spatial resolutions. It neither provides several bit-rates for a specific spatio-temporal resolution. On the other hand, SVC provides subset lower quality bit streams from a high quality video bit stream. This is done by dropping packets from the larger video so that the bandwidth required to send the subset bit stream is lower. These subset bit streams are referred as layers, and generally there is a Base Layer (BL) and one or more than one Enhancement Layers (EL).

In this work as we are dealing with UGC, the computation required to generate media segments for DASH remains an issue. Due to the amount of computation power required and limited memory of the UE, it is not practical to use H.264/AVC to generate multiple single layer streams of different bit rates and to store them at the UE. In comparison SVC provides the benefit of reduced computation power and less memory requirement to store segments.

\begin{tabular}{|c|c|c|}
\hline $300 \mathrm{kbps}$ & Segment 1 & Segment $\mathrm{N}$ \\
\hline $200 \mathrm{kbps}$ & Segment 1 & Segment $\mathrm{N}$ \\
\hline $100 \mathrm{kbps}$ & Segment 1 & Segment $\mathrm{N}$ \\
\hline
\end{tabular}

Single Layer Advance Video Coding

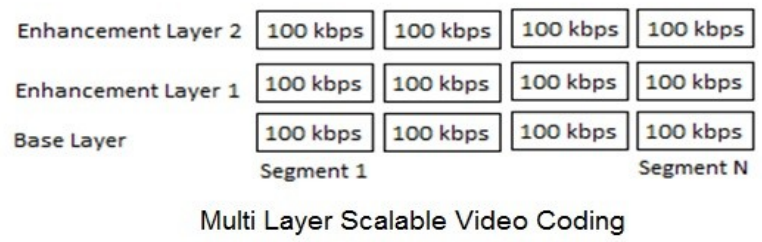

Figure 1: Comparison of AVC and SVC in terms of storage requirement

Figure 1 compares an example of SVC and H.264/AVC in terms of storage required. It shows, for AVC, a single segment of one second of video length needs a total of $(100+200+300)$ Kbits of storage. This will provide three different bit rate representations of $100 \mathrm{Kbps}, 200 \mathrm{Kbps}$ and $300 \mathrm{Kbps}$. The encoding is done independently for each representation that requires more computation and more battery consumption. On the other hand, when using SVC, a single segment of one second of video length needs a total of $(100+100+100)$ Kbits of storage. The three layers that provide different qualities are named as base layer, enhancement layer 1 and enhancement layer 2. If the client requests for lowest quality then only base layer (100 Kbits) is transferred. Whereas, if it requests for a better quality then enhancement layer 1 (100 Kbits +100 Kbits), i.e. $200 \mathrm{Kbits}$ in total, is transferred. In this way multiple streams at different bit rates are accessible, minimizing the total computation required at the UE and its battery utilization. Therefore, the selected video coding scheme for the implementation done in this work is SVC.

We decompose a video stream into three video layers where the base layer (BL), encoded at $15 \mathrm{fps}$, provides a basic video quality and the remaining enhancement layers (EL1 and EL2), encoded at $30 \mathrm{fps}$, provide a refined video quality. Figure 2 shows the Rate-MOS utility curves generated for different test videos we use in our simulation.

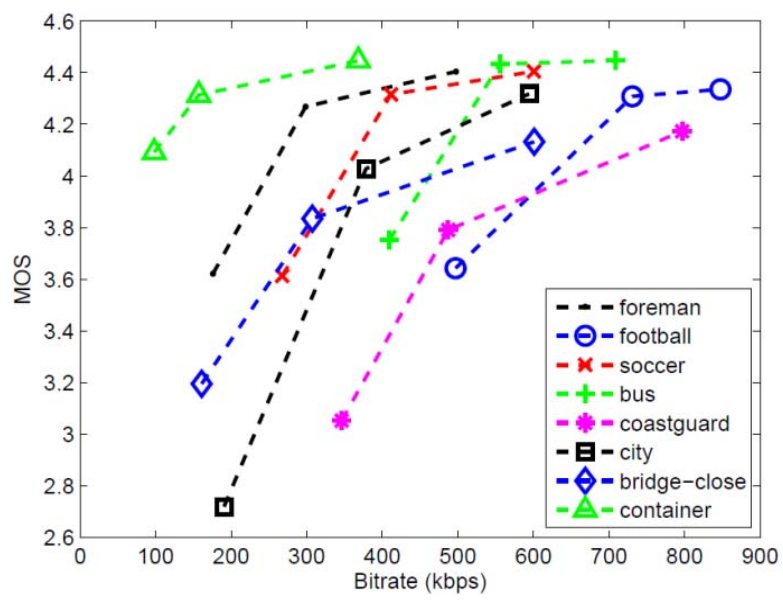

Figure 2: Video Streams Generated (Rate-MOS Utility Curves)

\section{B. Related Work}

Research work on resource allocation for DASH video streams has largely been focused on downlink. For example in [7] Wei Pu et. al presented a wireless proxy approach for DASH, WiDASH proxy, in order to maximize the viewers QoE. WiDASH proxy locates at the edge between Internet and wireless core networks. Different from conventional DASH whose rate adaptation logics are either implemented locally in the user equipment or in the DASH server, WiDASH proxy is in charge of video adaptation, which makes it feasible to perform global optimization over multiple concurrent DASH flows. Another example of resource allocation for DASH downlink streams is proposed in [8] in which an over the top (OTT) approach is used that requires no adaptation of the media content. The advantage of the proposed scheme is obvious in terms of gain in QoE in comparison to both reactive QoEoptimized and to standard-DASH HTTP streaming. 
For uplink user generated content, but that only for UDP/RTP based streaming, some work has been done by El Essaili et. al in [9] in which the proposed approach assigns more resources for popular contents while maintaining a minimum guaranteed QoE for the less popular ones. The results show that a significant gain in terms of average user satisfaction can be achieved.

\section{NETWORK-CENTRIC APPROACH}

SVC decomposes a video stream into three video layers where the base layer (BL) provides a basic video quality and the remaining enhancement layers (EL1 and EL2) provide a refined video quality. We consider time-shifted up streaming of scalable video from a video producer to a video portal which acts as an intermediate node for archiving of video streams for on-demand retrieval. The uplink UEs are the video producers. They have the SVC streams generated that are available for being transmitted over the radio access network. The eNodeB acts as a centralized resource allocation entity that decides on the resources to be provided to the video producers. The video content that is uploaded by the video producers is first stored on a video portal. This uploaded content is then accessed, from the video portal, by the viewers.

A schematic overview of a system for uplink distribution of user-generated content, from a set of video producers to a video portal, to be viewed on-demand is illustrated in figure 3 .

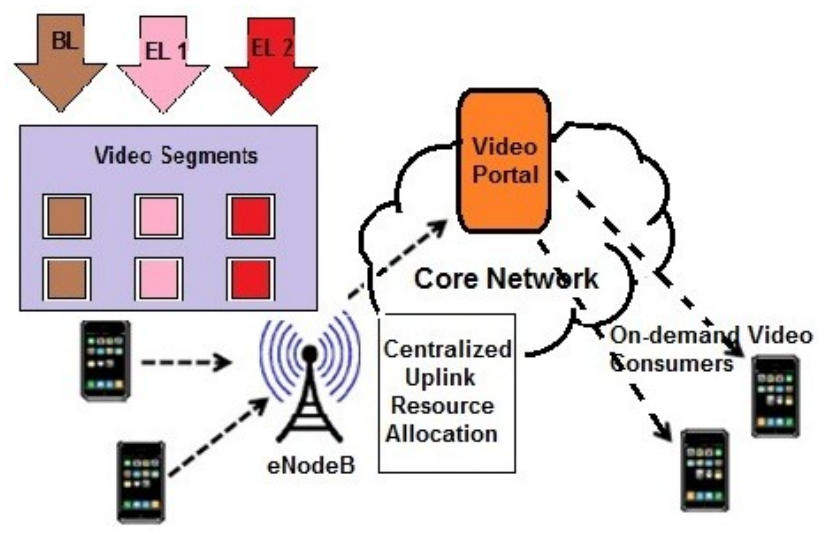

Figure 3: System Image for QoE-driven Uplink Resource Allocation

\section{QoE OPTMIZED RESOURCE ALLOCATION}

\section{A. LTE Uplink Model}

The link layer model used for uplink is recommended by 3GPP LTE in Release 8 [10]. It determines the achievable throughput per PRB for a given Signal-to-Noise ratio $(\gamma)$. The model approximates the throughput in the uplink, after link adaptation and hybrid automatic repeat request, by an implementation loss $\beta=0.4$ compared to the Shannon capacity, as defined in equation 1. It further defines range of uplink parameters with $\gamma_{\min }=-10 \mathrm{~dB}, \gamma_{\max }=-15 \mathrm{~dB}$ and a maximum throughput $\left(\mathrm{Thr}_{\max }\right)$ of $2 \mathrm{bps} / \mathrm{Hz}$.

Thr $=\left\{\begin{array}{cl}0 & \text { for } \gamma<\gamma_{\min } \\ \beta \log _{2}(1+\gamma) & \text { for } \gamma_{\min } \leq \gamma \leq \gamma_{\max } \\ \operatorname{Thr}_{\max } & \text { for } \gamma>\gamma_{\max }\end{array}\right.$

\section{B. Resource Allocation Framework}

As we are working with DASH videos that are encoded using scalable video coding, the number of different video quality representations for the streams are limited. In our case we only have three SVC layers, that means we have less flexibility when trying to optimize the resource distribution between the uplink video producers in order to maximize the MOS of the viewers. We introduce a gradient based resource allocation algorithm that provides discrete optimization which is more suitable to limited number of data rate representations. The objective function is defined in equation 2 .

subject to

$$
\underset{\left(\alpha_{1}, \alpha_{2}, \ldots \alpha_{K}\right)}{\arg } \max \sum_{\mathrm{k}=1}^{\mathrm{K}} \mathrm{U}_{\mathrm{K}}\left(\alpha_{\mathrm{K}}\right)
$$

$$
\sum_{\mathrm{k}=1}^{\mathrm{K}} \alpha_{\mathrm{K}}<=1
$$

The objective of the uplink resource allocation is to determine the resource share $\alpha_{K}$ of each user that maximizes the overall QoE. For the given resource share, the utility function of user $\mathrm{k}$ is denoted by $\mathrm{U}_{\mathrm{K}}$. The inequality in the constraint is due to the limited number of SVC layers. As we only use three RateMOS representations available on the utility curve in order to achieve the objective function defined, we may end up with few physical resource blocks not assigned if they are not enough to obtain the next higher rate representation.

The gradient based resource allocation algorithm, determines the resource share of every video producer. It starts by first assigning the resources, to all video producers, that are enough to transmit their base layers. After that, in order to send the enhancement layer, the algorithm then assigns the resources to the producer whose video provides the maximum gradient on the utility curve. Here the gradient is defined as the increase in utility with respect to the amount of resources required for that increase. The algorithm runs assigning resources to the next video producer that has the maximum gradient on the utility curve. It stops if all resources are utilized or when EL2 of all video producers is transmitted or if the remaining resources are not sufficient to get the next higher layer of any of the videos. 


\section{SIMULATION RESULTS}

\section{A. Scenario}

The simulation model that has been implemented for ondemand uplink DASH was described in section III. Here we assume that there is no delay or congestion experienced by the packets over the core network. Furthermore, we consider a single LTE cell with one eNodeB. The number of video producers uploading the video content is 8 and so as the number of viewers. All the video producers upload different videos that are available in three different qualities (base layer, enhancement layer 1, enhancement layer 2). The number of optimization cycles is 100 and the total simulation time is 100 seconds.

\section{B. Rate Adaptation Algorithm for DASH}

Here we also implement a receiver-driven rate adaptation algorithm, as defined in [11], which gives a more realistic QoE perspective of DASH viewers. This algorithm is based on the segment fetch time, and not on instantaneous TCP transmission rate, that decides on the data rate representation to be requested for the next DASH segment. The client measures the segment fetch time to determine if the bitrate of the current representation matches the available end-to-end bandwidth capacity. The segment fetch time (SFT) denotes a period of time from the time instant of sending a GET request for a media segment to the instant of receiving the last bit of the requested media segment. The client switches up the request rate if SFT is less than or equal to 1 segment, or in this case $1 \mathrm{sec}$ when the segment duration is itself $1 \mathrm{sec}$, and buffered media time is larger than the predefined minimum. Whereas the switch down takes place if SFT is greater than 1 segment. There is a complementary switch down if buffered media time is lower than the predefined minimum to prevent client buffer under flow. From here we state this rate adaptation as DASH SFT.

\section{On-demand Simulation Results}

In this section we evaluate the QoE-driven gradient based algorithm, with a classical round robin (RR) scheduling. Figure 4 shows the comparison between the average MOS of QoEdriven algorithm and RR scheduling. The pink curve shows the theoretical QoE optimized average MOS using the gradient algorithm, whereas the black curve shows the theoretical average MOS achieved by using RR scheduling. We also analyze the SFT-based rate adaptation algorithm here which gives a more realistic QoE perspective of DASH viewers. The blue curve shows the average MOS of the viewers for QoE optimized SFT scenario, whereas the red one shows the average MOS of the viewers for RR scheduling based SFT scenario.

The results show the advantage of our QoE scheme in comparison to RR scheduling, both theoretically and in case of a more practical rate adaptation (SFT-based).

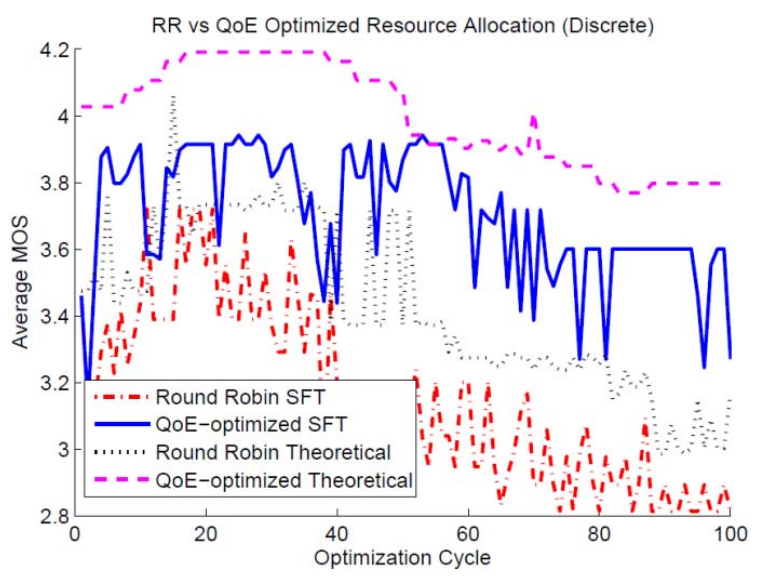

Figure 4: Average MOS for QoE gradient-based algorithm and RR scheduling

Figure 5 shows the comparison between the cumulative distribution function (CDF) for mean MOS of the QoE gradientbased algorithm and RR scheduling.

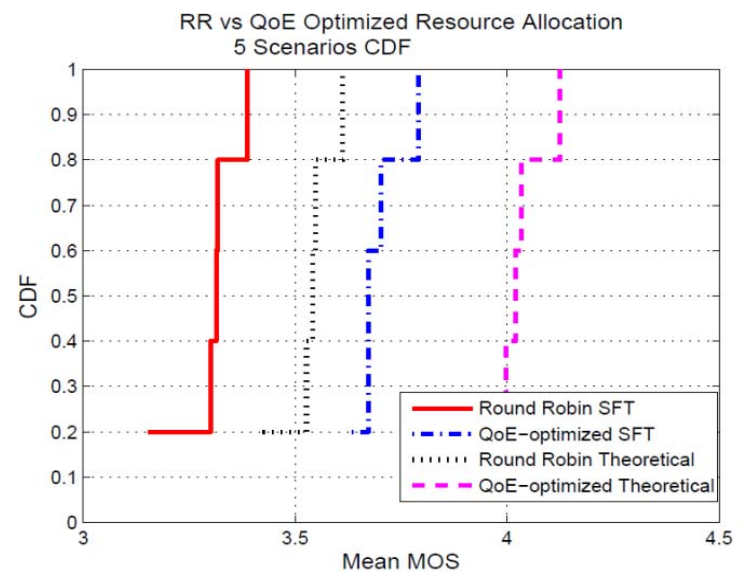

Figure 5: CDF for mean MOS of QoE gradient-based algorithm and RR scheduling

It confirms the advantage in terms of average MOS of our QoE-based resource allocation in comparison to RR scheduling. As we can see, the gain in average MOS is 0.5 for both theoretical and in case of rate adaptation (SFT-based) perspectives. In this section the number of scenarios used to 
generate the $\mathrm{CDF}$ is five, where the video producers experience different channel qualities in every different scenario.

Figure 6a shows the comparison between a SFT-based QoEbased scenario and a SFT-based RR scheduling scenario in terms of total number of buffered video segments at the viewers' end. It shows the buffer build-up for RR scheduling scenario although there is no gain in average MOS, hence unnecessarily increasing the uplink network load. Along with it, for the same scenario, figure $6 \mathrm{~b}$ shows the average channel quality for all the uplink video producers. We can see that the total buffer depends on the uplink channel quality of the video producers.

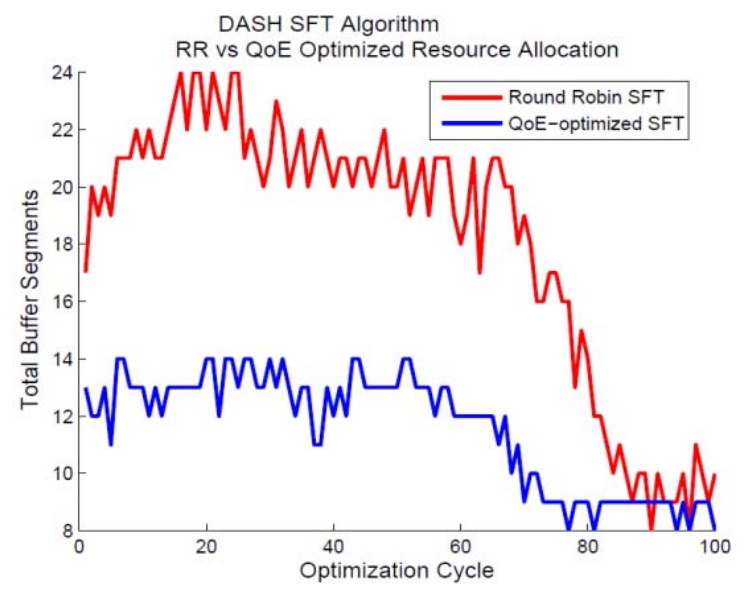

a) Total Buffer in segments for rate adaptation algorithm

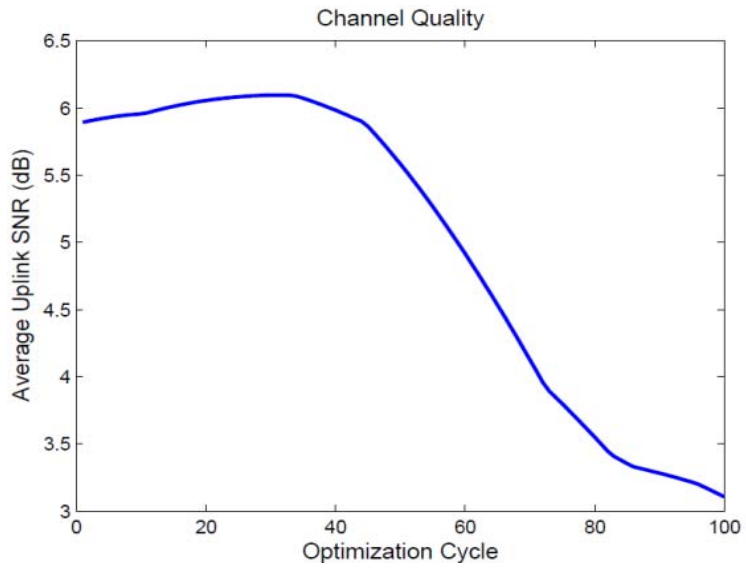

b) Average channel quality for all the uplink video producers

Figure 6: Comparison between a SFT-based QoE-based scenario and a SFTbased RR scheduling

\section{CONCLUSION}

We focused on optimized resource allocation for transmitting multiple DASH video streams in a LTE cell in the uplink direction. First, taking into account the challenges that can be faced for the video producers, DASH video streams that can be supported on LTE uplink air interface were generated. Then, an algorithm was designed that allocates physical resources to the video producers such that the QoE for the on-demand viewers is maximized. Simulation results were presented for LTE uplink resource distribution that demonstrate the significance of the proposed optimization on the overall user experience.

In future we look forward to implement a de-centralized approach for the network-based QoE optimization that can reduce exchange of information between the video producers and the eNodeB and thus reduce the overall optimization complexity.

\section{REFERENCES}

[1] 3GPP, TS 36.300 V10.5.0; Evolved Universal Terrestrial Radio Access (E-UTRA) and Evolved Universal Terrestrial Radio Access Network (EUTRAN); Overall description, Sep. 2011.

[2] Cisco, "Cisco Visual Networking Index: Global Mobile Data Traffic Forecast Update, 2012-2017," Feb. 2013.

[3] Sandvine Intelligent Broadband Networks. Sandvine, Global Internet Phenomena Report 1H 2013. Technical report, 2013.

[4] Thomas Stockhammer. DASH, Standards and Design Principles. In Proceedings of the second annual ACM conference on Multimedia systems, pages 133-144. ACM, 2011.

[5] Thomas Wiegand, Gary J Sullivan, Gisle Bjontegaard, and Ajay Luthra. Overview of the H. 264/AVC video coding standard. Circuits and Systems for Video Technology, IEEE Transactions, 13(7):560576, 2003.

[6] Heiko Schwarz, Detlev Marpe, and Thomas Wiegand. Overview of the scalable video coding extension of the H. 264/AVC standard. Circuits and Systems for Video Technology, IEEE Transactions on, 17(9):1103-1120, 2007.

[7] Wei Pu, Zixuan Zou, and Chang Wen Chen. Video adaptation proxy for wireless Dynamic Adaptive Streaming over HTTP. In Packet Video Work-shop (PV), 2012 19th International, pages 65-70. IEEE, 2012.

[8] Ali El Essaili, Damien Schroeder, Dirk Staehle, Mohammed Shehada, Wolfgang Kellerer, and Eckehard Steinbach. Quality-ofExperience driven Adaptive HTTP Media Delivery. In IEEE International Conference on Communications (ICC 2013), Budapest, Hungary, Jun 2013.

[9] Ali El Essaili, Eckehard Steinbach, Daniele Munaretto, Srisakul Thakolsri, and Wolfgang Kellerer. QoE-driven resource optimization for user generated video content in next generation mobile networks. In IEEE International Conference on Image Processing (ICIP 2011), Brussels, Belgium, Sep 2011.

[10] 3GPP TR 36.942 V.8.1.0. Evolved Universal Terrestrial Radio Access (EUTRA); Radio Frequency (RF) system scenarios. Technical report, Dec 2008.

[11] Chenghao Liu, Imed Bouazizi, and Moncef Gabbouj. Rate adaptation for adaptive HTTP streaming. In Proceedings of the second annual ACM conference on Multimedia systems, pages 169-174. ACM, 2011. 